\title{
Association between hippocampal subfields and clinical symptoms of first-episode and drug naive schizophrenia patients during 12 weeks of risperidone treatment
}

\section{XiaoE Lang}

Shanxi Medical University

\section{Dongmei Wang}

University of Chinese Academy of Sciences

\section{Dachun Chen}

Beijing Huilongguan hospital

\section{Mei Hong Xiu}

beijing huilongguan hospital

\section{Huixia Zhou}

CAS Key Laboratory of Mental Health, Institute of Psychology, Chinese Academy of Sciences; Department of Psychology, University of Chinese Academy of Sciences, Beijing, China

\section{Li Wang}

CAS Key Laboratory of Mental Health, Institute of Psychology, Chinese Academy of Sciences https://orcid.org/0000-0002-1459-3412

\section{Bo Cao}

University of Alberta, Department of Psychiatry https://orcid.org/0000-0001-9338-3271

Xiangyang Zhang ( $\square$ zhangxy@psych.ac.cn) Institute of Psychology, Chinese Academy of Sciences, Beijing, China; University of Chinese Academy of Sciences, Beijing, China https://orcid.org/0000-0003-3326-382X

\section{Article}

Keywords: Schizophrenia, hippocampus, first-episode, risperidone, treatment, prediction

Posted Date: August 13th, 2021

DOl: https://doi.org/10.21203/rs.3.rs-800348/v1

License: (9) (1) This work is licensed under a Creative Commons Attribution 4.0 International License. Read Full License

Version of Record: A version of this preprint was published at Neurotherapeutics on January 31st, 2022. See the published version at https://doi.org/10.1007/s13311-021-01174-8. 


\section{Abstract}

Small hippocampal size, especially in CA1 may be implicated in the pathogenesis and psychopathology of schizophrenia (SCZ). However, does the volume of hippocampal subfields like CA1 in SCZ patients affect response to antipsychotic treatment? In this study, we used risperidone to treat first-episode and drug naïve (FEDN) SCZ patients for 12 weeks, and then explored the relationship between differences in baseline hippocampal subfield volumes, as well as any changes in the volumes of these hippocampal structures during treatment, and improvement in their psychopathological symptoms. By adopting a state-of the-art automated algorithm, the hippocampal subfields were segmented in 43 FEND SCZ inpatients at baseline and in 26 of them after 12 weeks of risperidone monotherapy, as well as in 30 matched healthy controls. We adopted the Positive and Negative Syndrome Scale (PANSS) to assess psychopathological symptoms in 43 SCZ patients at baseline and in 40 of them at post-treatment. Our results showed that before treatment, schizophrenia patients had no significant differences in total or subfield hippocampal volumes compared with healthy volunteers (all $p>0.05$ ). However, at baseline smaller volumes of the left CA1, right molecular layer of the dentate, left hippocampal tail and left pre-subiculum were significantly correlated with worse PANSS negative symptom scores, accounting for $41 \%$ of its variance. We also found a significant correlation between a larger left CA1 at baseline and a worse PANSS total score and general psychopathology sub-score at post-treatment (both $p<0.05$ ). Furthermore, the left CA1 at baseline was significantly smaller in responders, who had $>50 \%$ improvement in PANSS total score, than in non-responders $(p<0.05)$. Our results suggest that FEDN SCZ patients with smaller left CA1 had greater negative psychotic symptoms at baseline and more of them had a $>50 \%$ improvement in PANSS total score after 12 weeks of risperidone treatment. Thus, smaller left CA1 volume may be a predicator for improvement in psychotic symptoms of FEDN SCZ patients, but hippocampal volumes overall did not differ in size from normal controls and did not change during treatment.

\section{Introduction}

Schizophrenia (SCZ) is one of the most common severe psychiatric disorders, with a prevalence rate around $1 \%$ worldwide ${ }^{1}$. Its core symptoms include positive symptoms, negative symptoms, and cognitive impairment. Although antipsychotic drugs can significantly improve the positive symptoms and some negative symptoms of patients, negative symptoms and cognitive impairment show limited responses ${ }^{2,3}$. The incomplete response to antipsychotics in some SCZ patients is not readily predicted, but Magnetic Resonance Imaging (MRI) technology can provide brain structure correlates for SCZ pharmacotherapy responses. In particular, many studies have demonstrated hippocampal volume reductions in SCZ patients' brains as the most distinguishing difference from healthy controls among both early and chronic patients ${ }^{4-10}$. Hippocampal abnormalities may be present before the first episode of psychosis 7,11 , and correlate with SCZ patients' clinical symptoms and cognitive impairment 9,12-16.

Anatomically, the formation of the hippocampus is not uniform, but consists of several subfields with distinct morphology and function, such as the cornu ammonis (CA) 1-4, dentate gyrus (DG), subiculum and presubiculum or entorhinal cortex (EC) ${ }^{17,18}$. Several MRI studies in chronic SCZ identified hippocampal subfields with smaller volumes including the CA1, left CA2/3 and CA4/DG and subiculum ${ }^{8,9,19}$. Moreover, the positive symptoms especially hallucinations and delusions were inversely correlated with the bilateral CA1 and CA2/3 subfield volumes in chronic SCZ patients ${ }^{20}$. One study showed a significant association between smaller pre- 
subiculum volumes and negative symptoms ${ }^{7}$. However, only a few studies reported on first episode SCZ patients, and one found lower CA1 and CA2 subfield volumes in the left hemisphere ${ }^{21}$, while recent studies demonstrated that there was no difference in the volumes of hippocampal subfields between first-episode SCZ patients and healthy volunteers ${ }^{9,22}$.

Some previous studies have shown that antipsychotic treatment may affect hippocampal volumes ${ }^{23,24}$ with the dose of antipsychotic drugs being inversely associated with total hippocampal and subfield volumes ${ }^{25,26}$. Another study demonstrated reduced CA1 volume in early SCZ, with this focal atrophy in early illness extending to $\mathrm{CA} 2 / 3^{9}$. In contrast, another recent study showed significantly larger subfield volumes in bilateral CA4 and bilateral molecular and granular cell layers of DG in FEDN SCZ patients, but without differences from controls in total hippocampal volume. After 6-week antipsychotic treatment, patients displayed volume decreases in total hippocampus, as well as several subfields including those previously enlarged subfields at baseline, which were decreased to the levels of healthy controls ${ }^{27}$. One interpretation is that antipsychotic treatment may correlate with hippocampal brain tissue loss over time ${ }^{23}$.

Based on this consensus of reduced hippocampal CA1 volume in chronic and perhaps FEDN SCZ patients and a potential antipsychotic role in reducing hippocampal brain tissue with greater antipsychotic doses, we examined the correlations between hippocampal subfield volumes particularly CA1 and clinical symptoms in FEDN SCZ patients during risperidone treatment. The present study had three purposes. 1) Whether the FEDN SCZ patients at baseline differed from controls in their hippocampal subfield volumes particularly in CA1 and whether those volumes correlated with baseline PANSS scales. 2) Whether baseline CA1 volumes would predict FEDN SCZ patients' responses to risperidone treatment. 3) Whether risperidone treatment would have a significant effect on the hippocampal subfields like CA1; and whether any changes in the hippocampal subfields would correlate with improvements in psychopathological symptoms.

\section{Results}

\section{Baseline comparisons between SCZ patients and healthy volunteers}

All patients and healthy controls were right-handed. We did not find significant differences in sex, age, and education between SCZ patients and healthy volunteers (all $p>0.05$ ). Also, we did not observe significant differences in any of hippocampal subfields between SCZ patients and healthy volunteers (all $p>0.05$ ).

\section{Relationships between psychopathology and the subfield volumes in SCZ at baseline}

Using multivariate regression analysis, larger volumes of the right molecular layer $(p=0.003)$, left CA1 $(p=0.008)$, left hippocampal tail $(p=0.014)$ and left pre-subiculum $(p=0.019)$ were significantly correlated with worse PANSS negative symptom scores at baseline, accounting for $41 \%$ of its variance.

\section{Changes in clinical symptoms and subfield volumes after treatment}

Of these 43 patients, 3 dropped out before 12-week treatment, which we did not include in the statistical analysis. The remaining 40 patients completed the full 12-week trial, and their demographics and clinical parameters are present in Table 1. After 12 weeks of risperidone treatment, all PANSS three subscale scores and total score were significantly reduced (all $p<0.05$; Table 1 ). 
After 12 weeks of treatment, we obtained the hippocampal subfield data of 26 patients, because 10 patients refused to receive the second MRI scan and 4 patients were unable to obtain an MRI scanner. After 12 weeks of risperidone treatment, we did not observe any significant changes in all hippocampal total and subfield volumes (all p>0.05; Table 2).

We found a significant correlation between a larger left CA1 at baseline and a worse PANSS total score ( $r=0.35$, $d f=40, p<0.05)$ and general psychopathology sub-score at post-treatment $(r=0.33, d f=40, p<0.05)($ Figure 1$)$. After controlling for ICV, sex, age, and age of onset as covariates, these correlations remained significant (both $p<0.05)$. However, these significances did not pass Bonferroni correction (both $p>0.05$ ). In addition, there were no significant correlations between the reduction of PANSS scores and changes in any hippocampal subfield volumes (all $p>0.05)$.

Further, we used the criteria of a $50 \%$ or more improvement in PANSS total score to define "responders" or "nonresponders". Compared to the non-responder group (623.3 $\pm 56.8 ; n=19)$, the responder group $(582.6 \pm 42.3 ; n=21)$ showed significantly lower left $C A 1$ volume at baseline $(F=4.25, d f=1,39, p<0.05)$. After controlling for the confounding factors, ANCOVA analysis still showed significant difference in left CA1 at baseline $(F=3.88, d f=6$, $33, p<0.05)$.

\section{Discussion}

This study found no significant hippocampal subfield volume deficits in FEDN SCZ patients compared to normal controls, but small volumes of some subfields at baseline were associated with greater negative symptoms. Furthermore, small left CA1 volume at baseline may predict the response to 12 weeks of risperidone treatment, especially for improved general psychopathological symptoms, although no hippocampal subfield volumes showed significant volume changes after treatment.

\section{Hippocampal subfields in FEDN SCZ patients at baseline}

Although many studies showed smaller volumes of the whole hippocampus or its subfields in SCZ ${ }^{4,6,7,9}$, we did not observe any hippocampal subfield volume deficits in the patients with FEDN SCZ, which is consistent with a previous study in patients with first episode SCZ, showing no significantly abnormal hippocampal subfields ${ }^{8}$. Some studies have indicated that a longer course of disease and prior antipsychotic treatment may contribute to volume reductions of hippocampal subfields in SCZ patients $23,24,26$. One recent study indicated that longer untreated psychosis duration was also correlated with smaller whole hippocampal and some of subfield volumes $^{28}$. Another study demonstrated that the CA1 volume was selectively decreased in patients in the early to mid-course of schizophrenia. Moreover, their longitudinal analysis demonstrated that the focal atrophy correlated with early disease extended beyond CA1 over time and involved other subfields, including $\mathrm{CA} 2 / 3$ and $\mathrm{GCL}^{9}$. Another longitudinal study showed that after 12-week antipsychotic treatment for patients with first-episode psychosis, DG and CA4 volumes were significantly reduced ${ }^{29}$. Another recent study showed significantly greater volumes of hippocampal subfields in FEDN SCZ patients at baseline, and after only 6-week treatment with antipsychotics, these patients exhibited volume reductions in total hippocampus and several subfields ${ }^{27}$. Our results suggest that acute or mid-term antipsychotic treatment may not impact hippocampal structural volume, 
although reduced hippocampal subfield volumes seem characteristic of chronic SCZ patients. Different antipsychotic drugs may also have different effects on hippocampal volumes, since our study used a monotherapy with risperidone at a relatively low to moderate dose of $3 \sim 6 \mathrm{mg} / \mathrm{day}$, while the patients in other studies took various types of antipsychotic drugs $9,27,29$. A previous animal study reflected such medication specific effects such that olanzapine administration caused significant reduction in total hippocampus, CA1 and DG molecular layer volumes ${ }^{30}$. Thus, these results show reduced volumes of hippocampal subfields in chronically treated SCZ patients, but the results are mixed for FEDN patients,

While a recent study reported higher hippocampal subfield volumes in patients with first-episode antipsychotic naïve SCZ ${ }^{27}$, another more recent study found more hippocampal subfield deficits (particularly right hippocampus) in never-treated than treated SCZ patients, suggesting that antipsychotic treatment may reduce

shrinkage of hippocampal structures in $\mathrm{SCZ}^{26}$. These discrepancies may reflect different methods of acquiring the neuroimaging data or segmenting the hippocampal subfields, different course of disease, exposure to antipsychotic treatment, or even different ethnicities. We speculate that the hippocampus and its subfields may be unimpaired at the onset of SCZ, but the hippocampus gradually becomes damaged and shrinks with the development of illness. Contributing factors may be long-term exposure to chronic duration of disease, antipsychotic treatment, smoking, antipsychotic-induced side effects or comorbid diseases.

We found that multiple hippocampal subfields including left CA1, right molecular layer, left pre-subiculum and left hippocampal tail were significantly correlated with PANSS negative symptoms, but few other studies have explored the relationship between clinical symptoms and hippocampal subfields of SCZ patients. A previous study showed that the posterior CA1 and left anterior subfield volume deficits were correlated with positive symptoms, especially hallucinations and delusions ${ }^{31}$. A subsequent study found that CA1 and CA2/3 subfield volumes were negatively associated with PANSS ${ }^{19}$. Another study showed smaller subiculum volumes were associated with negative symptoms ${ }^{7}$. Taken together with our findings, we propose that structural damage to the hippocampus is not evident at SCZ onset across all patients, but patients with smaller hippocampal subfields may have more severe negative symptoms, possibly due to abnormal functional activity in these subfields such as left CA1. Interestingly, a previous study reported that the right hippocampus of patients with first-episode SCZ had greater resting state activity, which was associated with more negative symptoms, perhaps reflecting the relatively smaller volumes and deficits in functional activity of their left hippocampal subfields ${ }^{32}$, as we found in the current study. Prolonged SCZ disease may lead to a progressive loss of hippocampal subfield volumes ${ }^{8}$, as we noted above, but shorter term treatment does not appear to shrink hippocampal volumes in FEDN SCZ patients.

\section{The left CA1 volumes at baseline may predict the response to risperidone treatment for general psychopathological symptoms}

We found no significant hippocampal subfield deficits in FEDN SCZ patients compared to normal controls, but smaller baseline hippocampal subfield volumes, especially of CA1 may predict improvement with risperidone treatment, specifically in general psychopathological symptoms. A recent report revealed that baseline right CA3 volume was positively correlated with improvement in Global Assessment of Functioning (GAF) after 6 weeks of antipsychotic treatment ${ }^{27}$, which is consistent with our current study when contrasting right to left sided hippocampal volumes (left relatively smaller). A previous study also showed that over a 6-year follow-up, a 
subgroup of SCZ patients exhibited an increase in bilateral hippocampal volumes, who had better results in clinical, cognitive and functional domains ${ }^{33}$. These findings indicate that the hippocampal volume at baseline may be a potential biomarker for antipsychotic treatment response in schizophrenia. However, hippocampal laterality may be important for the optimal use of this non-invasive biomarker to predict treatment response in early schizophrenia.

In this study, there are several limitations that need to be mentioned. First, although considering the inherent challenges of recruiting FEDN patients, our sample size in this study was still relatively small, so replicated research samples from different races will be important to confirm our results. Second, in our current study, only a single antipsychotic drug risperidone at a relatively stable dose was used. Therefore, the dose correlation must be carefully considered, and whether the results of this study can be generalized to other antipsychotics and other higher doses requires further research. Third, the treatment duration of this study in FEDN schizophrenia is relatively short, and the effect of long-term antipsychotic treatment on the hippocampal subfields needs to be explored in the future.

In summary, we did not observe obvious baseline hippocampal subfield deficits in patients with FEDN SCZ; however, patients with larger hippocampal subfields may have more severe negative symptoms. We found that the baseline smaller left CA1 was significantly corrected with the post-treatment PANSS general psychopathology and total scores after 12 weeks of risperidone treatment, suggesting that the smaller baseline left CA1 may predict the response to risperidone treatment. However, we found no significant association between changes in hippocampal subfield volumes and improvement in clinical symptoms of SCZ patients after 12 weeks of treatment. Overall, due to the relatively limited sample size, using a single antipsychotic risperidone at a relatively modest and stable dose, and a relatively short treatment duration, replicated research samples from different ethnicities using different antipsychotic treatments for a long time will be optimal for supporting use this biomarker with antipsychotic treatment and prognosis. .

\section{Methods}

\section{Subjects}

A total of 43 FEDN inpatients were enrolled from the Beijing Hui-Long-Guan Psychiatric Hospital. The inclusion criteria included: (1) meeting DSM-IV criteria for a SCZ diagnosis, made by two independent psychiatrists using the Structured Clinical Interview for DSM-IV (SCID); (2) an acute episode; (3) age between 16 and 45 years, Han Chinese; (4) course of disease $\leq 5$ years; (5) no previous psychotropic drug treatment.

We randomly recruited 30 healthy controls with ages ranging from 16 to 45 years from a community in Beijing during the same period. Under the supervision of a research psychiatrist, trained researchers interviewed them. They did not have a personal history of Axis I disease, assessed by the researchers using SCID, and their firstdegree relatives did not have any known history of mental illness. The researchers used SCID for the assessment. They did not have a personal history of Axis I disease, and their first-degree relatives did not have any known history of mental illness.

All participants underwent physical examination and laboratory tests. Exclusion criteria for the participants were (1) having current physical diseases; (2) personal and family history of any brain diseases; (3) lifetime alcohol or substance abuse/dependence history except for tobacco; (6) refusal to provide written informed consent. 
The Institutional Review Committee of Beijing Huilongguan Hospital approved the research protocol. We obtained the written informed consent of each participant.

\section{Study design and Assessments}

Risperidone was used to treat 43 patients for 12 weeks. During the first week, the risperidone dose was titrated to $3 \sim 6 \mathrm{mg}$ per day and remained at these levels throughout the study period. Also, lorazepam or chloral hydrate was used for insomnia, and benzhexol hydrochloride (as needed) for extrapyramidal side effects. There was no other prescribed drug during the study.

By using the Positive and Negative Syndrome Scale (PANSS), two psychiatrists assessed the patient's psychopathological symptoms while they were unaware of the patients' status. They both simultaneously received a training session in using PANSS before this study began. After training, they maintained an inter-rater correlation coefficient more than 0.8 for the PANSS total score during repeated assessments. They rated all patients at baseline and at the end of 12-week treatment. For each patient, the same investigator rated these scales at baseline and at 12 weeks.

\section{Acquisition and pretreatment of MRI data}

The GE 3 Tesla MRI scanner was used to acquire the structural T1 weighted scan of each participant. Then the spoiled gradient echo (SPGR) sequence was adopted with these parameters: repetition time $(T R)=6.2 \mathrm{~ms}$, echo time $(T E)=2.8 \mathrm{~ms}$, slice thickness $=1.2 \mathrm{~mm}$, matrix size $=256 \times 256$ and 142 slices, field of view $(F O V)=240$ $\mathrm{mm}$, and flip angle $=8^{\circ}$. We used FreeSurfer software to perform subcortical segmentation ${ }^{34}$. An automated algorithm was employed to segment hippocampal subfields ${ }^{35}(29)$.

A subfield segmentation map was established as reported our previous studies ${ }^{13,35}$. In this study, there were 8 hippocampal subfields including: CA1 CA4, GCL, ML, pre-subiculum, subiculum, and the hippocampal tail ${ }^{35}$.

\section{Statistical Analyses}

Initial analysis included all patients and healthy controls. Normality of the data was analyzed by the KolmogorovSmirnov test. $\mathrm{x}^{2}$ test and one-way analysis of variance were performed to compare group differences. Further, after controlling for covariates such as intracranial volume (ICV), age, sex, and education, one-way analysis of covariance (ANCOVA) was employed to compare each hippocampal subfield between patients and healthy volunteers. Then, using ICV, age, and gender as covariates, a partial correlation was performed between general clinical data, clinical symptoms on PANSS scores, and hippocampal subfield volumes. Further stepwise multiple regression analysis was conducted to confirm the relationship among clinical variables, clinical symptoms and hippocampal subfields.

The effect of treatment was assessed using repeated measures multivariate analysis of variance (MANOVA) with the PANSS scores and the hippocampal subfield volumes as outcome measures. Then we performed partial correlation analysis to explore the relationship between symptom improvement and hippocampal subfield volume changes, with ICV, sex, age, and age of onset as covariates. We employed Bonferroni correction for multiple tests. Finally, exploratory regression analyses was carried out to investigate whether there were associations between changes in volumes of hippocampal subfields and improvement in clinical symptoms. 
In addition, we used the criteria of a $50 \%$ or more improvement in PANSS total score to define "responders" or "non-responders". We examined whether risperidone treatment for 12 weeks altered the hippocampal subfield volumes and whether differences in the hippocampal subfields occurred between responders and nonresponders.

All statistical analyses were carried out using SPSS version 18.0 (SPSS Inc., Chicago, United States), and the significance level was $p<0.05$ (two-tailed).

\section{References}

1. Simeone JC, Ward AJ, Rotella P, Collins J, Windisch R. An evaluation of variation in published estimates of schizophrenia prevalence from 1990-2013: a systematic literature review. BMC Psychiatry 15, 193 (2015)

2. Ventriglio A, Bellomo A, Ricci F, Magnifico G, Rinaldi A, Borraccino L, et al. New Pharmacological Targets for the Treatment of Schizophrenia: A Literature Review. Curr Top Med Chem. doi:

$10.2174 / 1568026621666210701103147$ (2021)

3. Lahteenvuo M, Tiihonen J. Antipsychotic Polypharmacy for the Management of Schizophrenia: Evidence and Recommendations. Drugs. doi: 10.1007/s40265-021-01556-4 (2021)

4. Adriano F, Caltagirone C, Spalletta G. Hippocampal volume reduction in first episode and chronic schizophrenia: a review and meta-analysis. Neuroscientist. 18, 180-200 (2012)

5. van Erp TG, Hibar DP, Rasmussen JM, Glahn DC, Pearlson GD, Andreassen OA, et al. Subcortical brain volume abnormalities in 2028 individuals with schizophrenia and 2540 healthy controls via the ENIGMA consortium. Mol Psychiatry. 21(4):585 (2016)

6. Mathew I, Gardin TM, Tandon N, Eack S, Francis AN, Seidman LJ, et al. Medial temporal lobe structures and hippocampal subfields in psychotic disorders: findings from the Bipolar-Schizophrenia Network on Intermediate Phenotypes (B-SNIP) Study. JAMA Psychiatry. 71, 769-777 (2014)

7. Haukvik UK, Westlye LT, Morch-Johnsen L, Jorgensen KN, Lange EH, Dale AM, et al. In vivo hippocampal subfield volumes in schizophrenia and bipolar disorder. Biol. Psychiatry. 77, 581-588 (2015)

8. Kawano M, Sawada K, Shimodera S, Ogawa Y, Kariya S, Lang DJ, et al. Hippocampal subfield volumes in first episode and chronic schizophrenia. PLoS One. 10, 1-14 (2015) 
9. Ho NF, Holt DJ, Cheung M, Iglesias JE, Goh A, Wang M, et al. Progressive Decline in Hippocampal CA1 Volume in Individuals at Ultra-High-Risk for Psychosis Who Do Not Remit: Findings from the Longitudinal Youth at Risk Study. Neuropsychopharmacology 42(6), 1361-1370 (2017)

10. Tamminga CA, Southcott S, Sacco C, Wagner AD, Ghose S. Glutamate dysfunction in hippocampus: relevance of dentate gyrus and CA3 signaling. Schizophr Bull. 38, 927-935 (2012)

11. Vargas T, Dean DJ, Osborne KJ, Gupta T, Ristanovic I, Ozturk S, et al. Hippocampal Subregions Across the Psychosis Spectrum. Schizophr Bull. 44(5), 1091-1099 (2018)

12. Tamminga CA, Stan AD, Wagner AD. The hippocampal formation in schizophrenia. Am J Psychiatry 167, $1178-1193$ (2010)

13. Xiu MH, Lang X, Chen DC, Cao B, Kosten TR, Cho RY, et al. Cognitive Deficits and Clinical Symptoms with Hippocampal Subfields in First-Episode and Never-Treated Patients with Schizophrenia. Cereb Cortex. 31(1), 89$96(2021)$

14. Krystal JH, Anticevic A, Yang GJ, Dragoi G, Driesen NR, Wang XJ, et al. Impaired tuning of neural ensembles and the pathophysiology of schizophrenia: a translational and computational neuroscience perspective. Biol Psychiatry. 81, 874- 885 (2017)

15. Lieberman JA, Girgis RR, Brucato G, Moore H, Provenzano F, Kegeles L, et al. Hippocampal dysfunction in the pathophysiology of schizophrenia: a selective review and hypothesis for early detection and intervention. Mol Psychiatry 23, 1764-1772 (2018)

16. McHugo M, Talati P, Armstrong K, Vandekar SN, Blackford JU, Woodward ND, et al. Hyperactivity and Reduced Activation of Anterior Hippocampus in Eary Psychosis. Am J Psychiatry 176(12), 1030-1038 (2019)

17. Kesner and Rolls. A computational theory of hippocampal function, and tests of the theory: new developments. Neurosci Biobehav Rev. 48, 92-147 (2015)

18. Nakahara S, Matsumoto M, van Erp TGM. Hippocampal subregion abnormalities in schizophrenia: A systematic review of structural and physiological imaging studies. Neuropsychopharmacol Rep. 38(4), 156-166 (2018) 
19. Sasabayashi D, Yoshimura R, Takahashi T, Takayanagi Y, Nishiyama S, Higuchi Y, et al. Reduced Hippocampal Subfield Volume in Schizophrenia and Clinical High-Risk State for Psychosis. Front Psychiatry. 12, $642048(2021)$

20. Kuhn S, Musso F, Mobascher A, Warbrick T, Winterer G, Gallinat J. Hippocampal subfields predict positive symptoms in schizophrenia: first evidence from brain morphometry. Transl Psychiatry. 2, e127 (2012)

21. Narr KL, Thompson PM, Szeszko P, Robinson D, Jang S, Woods RP, et al. Regional specificity of hippocampal volume reductions in first-episode schizophrenia. Neuroimage 21, 1563-1575 (2004)

22. Wannan CMJ, Cropley VL, Chakravarty MM, Van Rheenen TE, Mancuso S, Bousman C, et al. Hippocampal subfields and visuospatial associative memory across stages of

schizophrenia-spectrum disorder. Psychol Med. 49(14), 2452-2462 (2019)

23. Ho BC, Andreasen NC, Ziebell S, Pierson R, Magnotta V. Long-term antipsychotic treatment and brain volumes: a longitudinal study of first-episode schizophrenia. Arch Gen Psychiatry. 68(2), 128-137 (2011)

24. Vernon AC, Natesan S, ModM, Kapur S. Effect of chronic antipsychotic treatment on brain structure: a serial magnetic resonance imaging study with ex vivo and postmortem confirmation. Biol Psychiatry. 69(10), 936$44(2011)$

25. Zierhut KC, Graßmann R, Kaufmann J, Steiner J, Bogerts B, Schiltz K. Hippocampal CA1 deformity is related to symptom severity and antipsychotic dosage in schizophrenia. Brain. 136(Pt 3), 804-814 (2013)

26. Hu N, Sun H, Fu G, Zhang W, Xiao Y, Zhang L, et al. Anatomic abnormalities of hippocampal subfields in never-treated and antipsychotic-treated patients with long-term schizophrenia. Eur Neuropsychopharmacol. 35, 39-48 (2020)

27. Li W, Li K, Guan P, Chen Y, Xiao Y, Lui S, et al. Volume Alteration of Hippocampal Subfields in First-Episode Antipsychotic-Naïve Schizophrenia Patients Before and After Acute Antipsychotic Treatment. Neuroimage Clin. 20, 169-176 (2018)

28. Briend F, Nelson EA, Maximo O, Armstrong WP, Kraguljac NV, Lahti AC. Hippocampal glutamate and hippocampus subfield volumes in antipsychotic-naive first episode psychosis subjects and relationships to duration of untreated psychosis. Trans/ Psychiatry. 10(1), 137 (2020) 
29. Rhindress K, Robinson DG, Gallego JA, Wellington R, Malhotra AK, Szeszko PR. Hippocampal subregion volume changes associated with antipsychotic treatment in first-episode psychosis. Psychol Med. 47(10), 17061718 (2017)

30. Barr AM, Wu CH, Wong C, Hercher C, Töpfer E, Boyda HN, et al. Effects of chronic exercise and treatment with the antipsychotic drug olanzapine on hippocampal volume in adult female rats. Neuroscience. 255, 147157 (2013)

31. Zierhut K, Bogerts B, Schott B, Fenker D, Walter M, Albrecht D, et al. The role of hippocampus dysfunction in deficient memory encoding and positive symptoms in schizophrenia. Psychiatry Res. 183(3), 187-194 (2010)

32. Tregellas JR, Smucny J, Harris JG, Olincy A, Maharajh K, Kronberg E, et al. Intrinsic hippocampal activity as a biomarker for cognition and symptoms in schizophrenia. Am J Psychiatry. 171(5), 549-556 (2014)

33. Lappin JM, Morgan C, Chalavi S, Morgan KD, Reinders AA, Fearon P, et al. Bilateral hippocampal increase following first-episode psychosis is associated with good clinical, functional and cognitive outcomes. Psychol. Med. 44, 1279- 1291 (2014)

34. Jovicich J, Czanner S, Greve D, Haley E, van der Kouwe A, Gollub R, et al. Reliability in multi-site structural MRI studies: effects of gradient non-linearity correction on phantom and human data. Neuroimage. 30, 436-443 (2006)

35. Cao B, Passos IC, Mwangi B, Amaral-Silva H, Tannous J, Wu MJ, et al. Hippocampal subfield volumes in mood disorders. Mol Psychiatry. 22, 1352-1358 (2017)

\section{Tables}

Table 1. Clinical symptoms at pre-and post-treatment in first-episode schizophrenia patients treated with risperidone for 10 weeks

\begin{tabular}{|c|c|c|}
\hline Pre-treatment & Post-treatment & $\mathrm{F}$ \\
\hline$(\mathrm{n}=43)$ & $(\mathrm{n}=40)$ & \\
\hline
\end{tabular}

\section{PANSS}

Positive subscale

Negative subscale

General Psychopathology

Total score
$25.2 \pm 6.8$

$21.2 \pm 9.4$

$43.8 \pm 14.0$

$90.6 \pm 24.8$
$15.6 \pm 7.4$

$17.7 \pm 8.0$

$29.8 \pm 12.1$

$63.1 \pm 24.8$
57.9

6.7

42.7

55.2

$$
<0.001
$$

$$
0.014
$$

$<0.001$

$<0.001$

Note: PANSS $=$ Positive and Negative Syndrome Scale 
Table 2. Hippocampal subfield volumes in first-episode schizophrenia patients at pre-and post-treatment with 10 weeks of risperidone treatment

\begin{tabular}{|c|c|c|c|c|}
\hline & Pre-treatment & Post-treatment & & \\
\hline & $(\mathrm{N}=43)$ & $(n=26)$ & $\mathrm{F}$ & $\mathrm{p}$ \\
\hline Left hippocampus & $3342.7 \pm 283.0$ & $3343.6 \pm 262.0$ & 0.001 & 0.99 \\
\hline CA1 & $604.7 \pm 60.5$ & $602.9 \pm 53.2$ & 0.03 & 0.86 \\
\hline CA3 & $196.3 \pm 18.6$ & $196.7 \pm 16.1$ & 0.02 & 0.90 \\
\hline CA4 & $251.2 \pm 24.9$ & $252.6 \pm 23.8$ & 0.10 & 0.76 \\
\hline GCL & $297.4 \pm 29.2$ & $297.7 \pm 27.5$ & 0.002 & 0.97 \\
\hline Presubiculum & $300.0 \pm 32.2$ & $299.6 \pm 28.6$ & 0.01 & 0.93 \\
\hline Subiculum & $400.0 \pm 39.1$ & $400.1 \pm 38.1$ & 0.05 & 0.83 \\
\hline Hippocampal tail & $526.9 \pm 72.9$ & $525.1 \pm 61.3$ & 0.04 & 0.85 \\
\hline Molecular layer & $543.6 \pm 48.8$ & $545.6 \pm 46.9$ & 0.06 & 0.82 \\
\hline Right hippocampus & $3357.8 \pm 275.0$ & $3357.6 \pm 222.4$ & 0.000 & 1.00 \\
\hline CA1 & $624.0 \pm 54.6$ & $624.8 \pm 45.6$ & 0.011 & 0.92 \\
\hline CA3 & $208.0 \pm 22.1$ & $207.4 \pm 19.7$ & 0.01 & 0.94 \\
\hline CA4 & $255.8 \pm 23.3$ & $257.5 \pm 21.9$ & 0.14 & 0.71 \\
\hline GCL & $301.0 \pm 26.8$ & $302.5 \pm 24.6$ & 0.09 & 0.74 \\
\hline Presubiculum & $282.4 \pm 36.1$ & $280.9 \pm 32.0$ & 0.12 & 0.73 \\
\hline Subiculum & $401.2 \pm 38.8$ & $399.0 \pm 32.0$ & 0.25 & 0.62 \\
\hline Hippocampal tail & $519.7 \pm 82.9$ & $519.2 \pm 66.6$ & 0.003 & 0.96 \\
\hline Molecular layer & $557.4 \pm 45.8$ & $557.0 \pm 38.9$ & 0.002 & 0.97 \\
\hline
\end{tabular}

Note: $\mathrm{CA}=$ cornu ammonis; $\mathrm{GCL}=$ granule cell layer.

\# There were no significant differences in hippocampal subfields between schizophrenia patients and healthy cont $\mathrm{p}>0.05)$.

Figures 

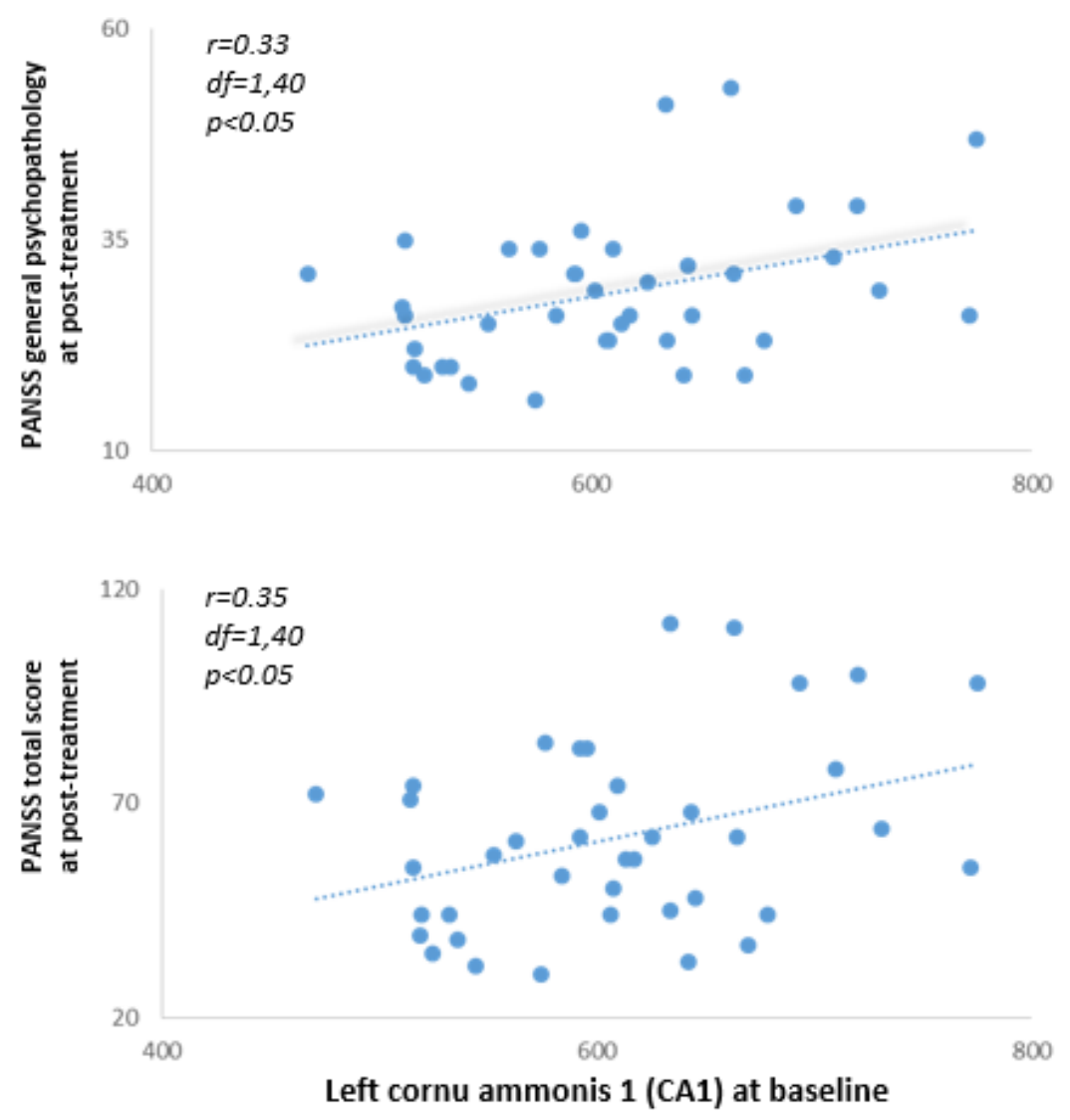

Figure 1

After controlling for sex, age, education, age of onset and intracranial volume (ICV), the partial correlation analysis showed that the volumes of left cornu ammonis 1 (CA1) at baseline was associated PANSS total score and general psychopathology (both $\mathrm{p}<0.05$ ) at the end of 10 weeks of risperidone treatment in first episode patients with schizophrenia. 